\title{
Effects of galactic dark halo rotation on WIMP direct detection
}

\author{
F. Donato ${ }^{\mathrm{a}, *}$, N. Fornengo ${ }^{\mathrm{a}, 1}$, S. Scopel ${ }^{\mathrm{b}, 2}$ \\ a Dipartimento di Fisica Teorica, Università di Torino, and INFN, Sezione di Torino, via P. Giuria 1, 10125 Torino, Italy \\ b Instituto de Física Nuclear y Altas Energías, Facultad del Ciencias, Universidad de Zaragoza, Plaza de San Francisco s/n. \\ 50009 Zaragoza. Spain
}

Received 16 March 1998; accepted 3 May 1998

\begin{abstract}
The effects of a possible rotation of the galactic dark halo on the calculation of the direct detection rates for particle dark matter are analyzed, with special attention to the extraction of the upper limits on the WIMP-nucleon scalar cross section from the experimental data. We employ a model of dark halo rotation which describes the maximal possible effects. For WIMP masses above $50 \mathrm{GeV}$, the upper limit exclusion plot is modified by less than a factor of two when rotation is included. For lighter masses the effect can be stronger, suggesting the necessity to develop specific models of halo rotation in order to provide more accurate conclusions. (c) 1998 Elsevier Science B.V.
\end{abstract}

\section{Introduction}

The possibility to detect Weakly Interacting Massive Particles (WIMPs) distributed in the halo of our Galaxy has been a major issue in the last years, since these particles could provide the amount of dark matter necessary to explain many observed dynamical properties of galaxies, clusters and of the Universe itself. Different kinds of possible signals have been identified and looked for, in order to outline the presence of WIMPs in our Galaxy. These signals are usually referred to as "direct" and "indirect" detection rates. Direct detection refers to the possibility to measure a WIMP-nucleus interaction in a low-background detector, while indirect detection relies on the measurement of WIMP annihilation products: photons, antiprotons and positrons produced by the annihilation in the galactic halo, or neutrinos coming out of the Earth or the Sun where WIMPs may have been accumulated as a consequence of gravitational capture. It is remarkable that the present sensitivity of the different experiments is already at the level of the predicted rates for specific WIMP candidates, like the neutralino, which represents one of the most interesting and studied cold relic particles [1].

The calculation of the different detection rates depends not only on the particle physics properties of the WIMPs interactions, but also on the characteristics of the galactic halo where the WIMPs are distributed. Direct

\footnotetext{
* Corresponding author. E-mail: donato@to.infn.it.

${ }^{1}$ E-mail: fornengo@to.infn.it.

${ }^{2}$ INFN Post-doctoral Fellow; scopel@posta.unizar.es.
} 
detection rates and upgoing-muon fluxes at neutrino telescopes, which both rely on the WIMP elastic scattering off nuclei, depend on the WIMP matter density $\rho_{\odot}$ and velocity distribution $f_{\odot}(v)$ at the Earth position $r_{\odot}$ in the Galaxy. In particular, the dependence of the signals on $\rho_{\odot}$ is a linear one. The other indirect signals (photon, antiproton and positron fluxes) have a stronger dependence on the matter distribution, since they are proportional to the square of the matter distribution function (DF) $\rho(\boldsymbol{r})$ integrated over the effective region of production and propagation of the annihilation products. On the contrary, this kind of signals are essentially independent on the details of the velocity DF, since the annihilating WIMPs are almost at rest and corrections due to their velocity dispersion are negligible.

Detailed estimates of the detection rates would require specific and accurate models of the galactic halo able to provide a reliable WIMPs DF $g(x, v)$ (not necessarily separable in phase space, i.e. $g(r, v)=\rho(\boldsymbol{r}) f(\boldsymbol{v})$ ). Unfortunately, detailed halo models are not available at present, mainly because the constraints obtained from astrophysical observations are not stringent enough to restrict different possibilities. The most important observational constraint is provided by the flatness of the rotation curves at large radii. Although the available data on our Galaxy do not provide a compelling evidence of a flat rotation curve, this feature is observed in a large number of spiral galaxies and therefore it looks reasonable to assume its validity also for our Galaxy.

The standard and simplest model of the dark galactic halo, which is compatible with a flat rotation curve, is the so-called isothermal sphere. This model relies on the two basic assumptions of spherical symmetry and thermal equilibrium, which find a strong support in the argument of "violent relaxation" introduced by LyndenBell 30 years ago [2]. In this model, the DF is separable into a matter density distribution $\rho(r)$, which has a $r^{-2}$ behaviour at large radii, and into a Maxwell-Boltzmann (MB) velocity DF $f(v)$ [3]. Although such a model gives a divergent total mass and therefore an appropriate cutoff has to be introduced at large radii, its range of validity has been tested at least in the inner parts of many galactic systems. Moreover, since it represents a simple and reasonable approximation, in the absence of a more detailed model it is widely adopted to describe the dark halo of our Galaxy. However, many different models are known to be consistent with flat rotational curves. For instance, models which describe nonspherically symmetric or flattened halo distributions have been discussed [3]. In these models, the specific form of $\rho(\boldsymbol{r})$ differs from the standard isothermal sphere matter DF, especially at small radii, entailing quite large uncertainties on the local value $\rho_{\odot}$. A comprehensive numerical study which takes into account a large number of models indicates that the local value of the nonbaryonic dark matter density falls in the (rather conservative) range $0.1 \lesssim \rho_{\odot} \lesssim 0.7 \mathrm{GeV} \mathrm{cm}^{-3}$ [4]. Contrary to the matter $\mathrm{DF}$, the specific form of the velocity DF $f(v)$ has been much less investigated. Modifications to the standard MB velocity DF are known [3,5], but the problem of determining the correct form of the distribution of the WIMP velocities in the halo has no clear and simple solution at present, both theoretically and observationally. The velocity DF is required to be consistent with a given $\rho(\boldsymbol{r})$ but this, in general, does not determine $f(v)$ in a unique way.

The calculation of the WIMP detection rates is usually performed by using the standard isothermal sphere model. However, modifications in the isothermal model can affect the detection rates, introducing uncertainties in the theoretical predictions and in the extraction of the experimental limits on the WIMPs parameters. The effects induced on the detection rates by a modification in the matter DF are simple to take into account, since the dependence of the detection rates on $\rho(\boldsymbol{r})$ can be factorized. Specifically, the physical range of $\rho_{\odot}$ quoted above implies an uncertainty of about a factor of 7 in the evaluation of the direct detection rates and in the neutrino fluxes [1] (it has to be remarked that this large factor reflects a rather conservative attitude). Even larger uncertainties affect the indirect rates from WIMP annihilation in the halo, since in this case a modification in the matter density profile can strongly affect the integral of $\rho^{2}(r)$ over the effective production region of the signal [6-8]. Contrary to the case of the matter DF, a modification of the standard MB velocity DF would affect the direct detection rates and the indirect rates at neutrino telescopes in a much more involved way. This is because the dependence of these rates on $f(v)$ is through a convolution of $f(v)$ with the differential WIMPnucleus cross section. Since the WIMP-nucleus scattering depends on the relative velocity of the WIMPs with respect to the detector nuclei, a potentially significant effect could be due to a bulk rotation of the halo. This 
would necessarily modify the WIMP phase-space DF with respect to the standard MB form.

In this paper we wish to discuss the possible effects induced by a halo rotation on the direct detection rates, with special attention to the ensuing consequences on the determination of the upper limits on the WIMPnucleus cross section from the experimental data. A calculation of the direct detection rates in the case of a rotating halo has been addressed in Ref. [9], where it has been concluded that the maximal effect of rotation leads to a $30 \%$ effect on the total detection rates for a Ge nucleus, in the case of an ideal detector with no threshold. However, when considering a real detector the behaviour of the differential rates at threshold and the detector characteristics are crucial in determining the experimental limits on the WIMP-nucleus cross section [1]. Therefore, we explicitly take into account the features of running detectors, such as thresholds, quenching factors and energy resolution, in order to estimate the largest uncertainties induced by a possible halo rotation in a confident way. To this aim, following Ref. [9] we model the galactic rotation as described by Lynden-Bell in Ref. [10], where the maximally rotating velocity DF compatible with a given mass distribution has been derived, on the ground of purely kinematical arguments. Even if Lynden-Bell's model of halo rotation may not represent a situation which is realized in a physical halo, we consider it useful to bracket the size of the effect of halo rotation on the direct detection rates.

The plan of our paper is the following. In Section 2 we briefly describe the calculation of the direct detection rates in the presence of halo rotation. In Section 3 we discuss our results for $\mathrm{Ge}, \mathrm{NaI}$ and Xe detectors, taking into account the most recent experimental data of the different Collaborations. Finally, in Section 4 we draw our conclusions. An appendix is added, where we report the analytical expressions of the relevant part of the direct detection rates which contain the details of the velocity DF in the case of the standard nonrotating, maximally co-rotating and maximally counter-rotating haloes.

\section{Direct detection rates}

The interaction of a WIMP of mass $m_{\chi}$ with a detector produces the recoil of a nucleus with energy $E_{R}$ of the order of few to tens $\mathrm{keV}$. The recoil energy can be measured by means of various experimental techniques with different nuclear species. At present, experiments are running with $\mathrm{Ge}, \mathrm{NaI}, \mathrm{Xe}, \mathrm{CaF}_{2}, \mathrm{TeO}_{2}$ detectors [11-20] and other nuclei are currently under investigation. The relevant quantity to be calculated and compared with the experimental measurements is the differential detection rate,

$$
\frac{d R}{d E_{R}}=N_{T} \frac{\rho_{\chi}}{m_{\chi}} \int d v f(v) v \frac{d \sigma}{d E_{R}}\left(v, E_{R}\right),
$$

where $N_{T}$ is the number of the target nuclei per unit of mass, $\rho_{\chi}$ is the local WIMP matter density, $v$ and $f(v)$ denote the WIMP velocity and velocity DF in the Earth frame $(v=|v|)$ and $d \sigma / d E_{R}$ is the WIMP-nucleus differential cross section. The nuclear recoil energy is given by $E_{R}=m_{\mathrm{red}}^{2} v^{2}\left(1-\cos \theta^{*}\right) / m_{N}$, where $\theta^{*}$ is the scattering angle in the WIMP-nucleus center-of-mass frame, $m_{N}$ is the nuclear mass and $m_{\text {red }}$ is the WIMPnucleus reduced mass. Eq. (1) refers to the situation of a monoatomic detector, like the Ge detectors. For more general situations, like for instance the case of $\mathrm{NaI}$, the generalization is straightforward.

The differential WIMP-nucleus cross section can be expressed as

$$
\frac{d \sigma}{d E_{R}}=\frac{\sigma_{0}}{E_{R}^{\max }} F^{2}(q)
$$

where $\sigma_{0}$ is the point-like total WIMP-nucleus cross section, $E_{R}^{\max }$ is the maximum value of $E_{R}$ and $F(q)$ denotes the nuclear form factor, expressed as a function of the momentum transfer $q^{2} \equiv|q|^{2}=2 m_{N} E_{R}$.

The nuclear form factor depends sensitively on the nature of the effective interaction involved in the WIMPnucleus scattering. To be definite, in the following we will consider the case of a WIMP-nucleus scalar inter- 
action, since this is the one which is currently accessible to the present sensitivity of running detectors $[1,21]$. In this case we use the Helm parameterization of the scalar form factor [22],

$$
F(q)=3 \frac{j_{1}\left(q r_{0}\right)}{q r_{0}} \exp \left(-\frac{1}{2} s^{2} q^{2}\right)
$$

where $s \simeq 1 \mathrm{fm}$ is the thickness parameter for the nucleus surface, $r_{0}=\left(r^{2}-5 s^{2}\right)^{1 / 2}, r=1.2 A^{1 / 3} \mathrm{fm}, A$ is the nuclear mass number and $j_{1}\left(q r_{0}\right)$ is the spherical Bessel function of index 1 .

In the isothermal halo model the velocity DF is a Maxwell-Boltzmann distribution in the galactic rest frame. Taking into account a finite escape velocity, its expression is the following:

$$
f_{\text {gal }}\left(v^{\mathrm{gal}}\right)=N\left(\frac{3}{2 \pi w^{2}}\right)^{3 / 2} \exp \left(-\frac{3\left(v^{\mathrm{gal}}\right)^{2}}{2 w^{2}}\right),
$$

where the normalization factor is

$$
N=\left[\operatorname{erf}(z)-\frac{2}{\sqrt{\pi}} z \exp \left(-z^{2}\right)\right]^{-1} .
$$

In the previous equations, $z^{2}=3 v_{\text {esc }}^{2} /\left(2 w^{2}\right)$ and $w$ denotes the root mean square velocity. In the isothermal sphere model, $w$ is related to the asymptotic value $v_{\infty}$ of the rotational velocities by the simple relation $w=\sqrt{\frac{3}{2}} v_{\infty}$. The measured rotational velocity of the Local System at the Earth's position is $v\left(r_{\odot}\right)=220 \pm$ $20 \mathrm{~km} \mathrm{sec}^{-1}$ [23] and remains almost flat (to roughly 15\%) between $4 \mathrm{Kpc}$ and $18 \mathrm{Kpc}$. Identifying this value with $v_{\infty}$ one gets the estimate $w=270 \pm 25 \mathrm{~km} \mathrm{sec}^{-1}$.

In order to evaluate the WIMP-nucleus interaction-rate, Eq. (4) has to be transformed to the rest frame of the Earth, which moves through the Galaxy with a velocity $v_{\odot}=232 \pm 20 \mathrm{~km} \mathrm{sec}^{-1}$ in the azimuthal direction (this value for $v_{\odot}$ takes into account the motion of the solar system with respect to the Local System). Therefore, the velocity $v$ of the WIMPs, as seen in the Earth's frame, is related to their velocity in the Galactic frame $v^{\text {gal }}$ by the following set of transformation equations: $v_{\phi}=v_{\phi}^{\text {gal }}-v_{\odot}$ in the azimuthal direction, and $v_{\perp}=v_{\perp}^{\text {gal }}$ and $v_{R}=v_{R}^{\mathrm{gal}}$ in the vertical $(\perp)$ and in the radial $(R)$ direction along the galactic plane.

By means of the previous definitions, the differential rate can be written in the form

$$
\frac{d R}{d E_{R}}=N_{T} \frac{\rho_{\chi}}{m_{\chi}} \frac{m_{N} \sigma_{0}}{2 m_{\text {red }}^{2}} F^{2}\left(q^{2}\right) \mathcal{I}\left(v_{\min }, v_{\odot}, v_{\text {esc }}\right),
$$

where the function $\mathcal{I}\left(v_{\min }, v_{\odot}, v_{\text {esc }}\right)$ contains all the details of the integration of the velocity DF $f(v)$ in the Earth's frame,

$$
\mathcal{I}\left(v_{\min }, v_{\odot}, v_{\mathrm{esc}}\right)=\int d v \frac{f(\boldsymbol{v})}{v}=\int_{v_{\min }\left(E_{R}\right)}^{v_{\mathrm{esc}}} v d v \bar{f}(v) .
$$

In Eq. (7) we have defined

$$
\bar{f}(v)=2 \pi \int_{(\cos \theta)_{\min }}^{(\cos \theta)_{\max }} d \cos \theta f(v, \cos \theta),
$$

where $(\cos \theta)_{\min }$ and $(\cos \theta)_{\max }$ depend on $v, v_{\odot}$ and $v_{\text {esc. }}$ In Eq. (7) $v_{\min }\left(E_{R}\right)=\left(m_{N} E_{R} /\left(2 m_{\text {red }}^{2}\right)\right)^{1 / 2}$. Moreover, we have explicitly considered that particles which possess velocities greater than the escape velocity 


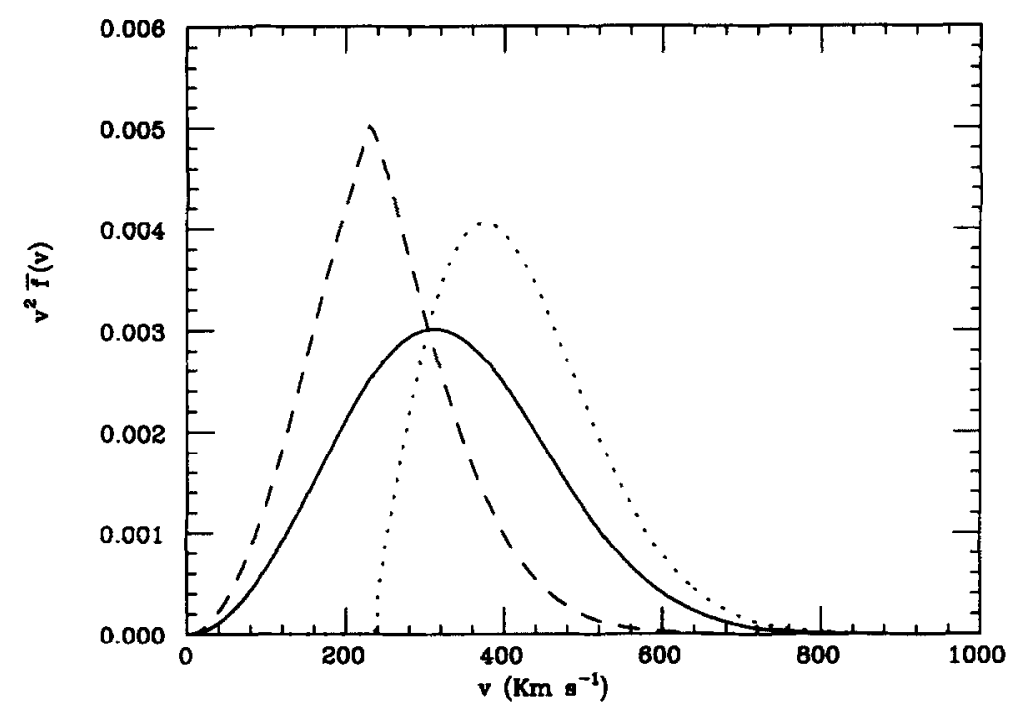

Fig. 1. Maxwell-Boltzmann velocity DF in the local frame (arbitrary units). Solid, dashed and dotted lines refer respectively to the nonrotating, maximal co-rotating and maximal counter-rotating case.

$v_{\text {esc }}$ are not bounded to the halo. The value of $v_{\text {esc }}$ is somewhat uncertain: $v_{\text {esc }}=600 \pm 200 \mathrm{~km} \mathrm{sec}^{-1}$ [24]. A low value of $v_{\text {esc }}$ can sizably affect the detection rates, especially at low WIMP masses. In the next section, we will use, as a reference value, $v_{\text {esc }}=650 \mathrm{~km} \mathrm{sec}^{-1}$.

Eq. (4) describes a galactic halo which does not possess a bulk rotation. In order to analyze the effect of a possible rotation of the isothermal sphere, we consider a class of models discussed by Lynden-Bell [10] which, for any given mass distribution, describe the fastest rotating steady state by means of the following recipe:

$$
\begin{aligned}
& f_{+}\left(v^{\mathrm{gal}}\right)= \begin{cases}f\left(v^{\mathrm{ga}}\right) & v_{\phi}^{\mathrm{gal}}>0, \\
0 & v_{\phi}^{\mathrm{gal}}<0,\end{cases} \\
& f_{-}\left(v^{\mathrm{gal}}\right)= \begin{cases}0 & v_{\phi}^{\mathrm{gal}}>0, \\
f\left(v^{\mathrm{gal}}\right) & v_{\phi}^{\mathrm{gal}}<0 .\end{cases}
\end{aligned}
$$

The LB model corresponds to an unrelaxed system with the maximal rotation compatible with a given mass distribution. There is no indication, neither theoretical nor observational, that this model can be realized in physical galactic haloes. Nevertheless, since it provides the largest rotation effect, we choose to use it in order to estimate the maximal modification induced by galactic rotation to the direct detection rates and to the extraction of the upper limits on the WIMP-nucleus cross section.

The analytic expressions for $\mathcal{I}\left(v_{\min }, v_{\odot}, v_{\text {esc }}\right)$ are given in the appendix for the nonrotating model of Eq. (4) and for the models of Eq. (9) (maximal co-rotation) and Eq. (10) (maximal counter-rotation). The three DFs $\bar{f}(v)$, for the nonrotating, co-rotating and counter-rotating cases, are plotted in Fig. 1. In the co-rotating situation the WIMPs have a bulk rotation in the same azimuthal direction as the Earth. Therefore, the relative velocity between the WIMPs and the detector is, on average, reduced. On the contrary, for a counter-rotating halo the relative average velocity increases and, moreover, there is a lower velocity cutoff corresponding to $v_{\odot}$. The reason why all the three curves in Fig. 1 cross at the same point is a feature of the particular choice of the distribution functions in Eqs. (9), (10) and does not reflect any general property of rotating models.

Eq. (6) represents the differential rate for an ideal detector. In order to compare the calculated rates with the measured ones, we have to express $\mathrm{Eq}$. (6) as a function of the electron-equivalent energy $E_{\mathrm{ee}}$ (which is 
Table 1

Threshold energy $E_{\mathrm{ee}}^{\mathrm{th}}$, quenching factor $Q$ and resolution parameters $a$ and $b$ for the detectors considered in the text

\begin{tabular}{llll}
\hline Detector & $E_{\mathrm{ee}}^{\text {th }}(\mathrm{keV})$ & $Q$ & Resolution parameters \\
\hline DAMA/NaI & 2 & Na: 0.31 & $\begin{array}{l}a=0 \\
b=0.579\end{array}$ \\
Ge/Neuchatel & 1.5 & I: 0.09 & $\begin{array}{l}a=0 \\
\end{array}$ \\
Ge/Twin & 4 & 0.25 & $b=0.17$ \\
& & & $a=0$ \\
DAMA/Xe & 13 & 0.25 & $b=0.05$ \\
& & 0.44 & $a=0.056$ \\
& & $b=1.191$
\end{tabular}

actually measured, instead of $E_{R}$ ) [25]. The quantity $E_{\mathrm{ee}}$ is simply proportional to the nuclear recoil energy through the quenching factor $Q$, i.e. $E_{\mathrm{ee}}=Q E_{R}$. Moreover, the energy resolution of the detector has to be taken into account. This is obtained by means of the convolution

$$
\frac{d R}{d E_{\mathrm{ee}}}=\frac{K}{\sqrt{2 \pi} r\left(E_{\mathrm{ee}}\right)} \int_{0}^{\infty} \exp \left(-\frac{\left(E_{\mathrm{ee}}-E\right)^{2}}{2 r^{2}\left(E_{\mathrm{ee}}\right)}\right) \frac{d R}{d E} d E,
$$

where $K$ is the normalization factor and is given by $K=2 /\left[1+\operatorname{erf}\left(E_{\mathrm{ee}} / \sqrt{2} r\left(E_{\mathrm{ee}}\right)\right)\right]$. The resolution function $r(E)$ can be expressed as a function of the energy as

$$
\frac{r(E)}{E}=a+\frac{b}{\sqrt{E}}
$$

where the energy $E$ is expressed in $\mathrm{keV}$. The constant parameters $a$ and $b$ depend on the detector and are determined experimentally. The values of the quenching factors $Q$ and of the resolution parameters $a$ and $b$ are reported in Table 1 for the detectors considered in Section 3. The threshold energies $E_{\mathrm{ee}}^{\text {th }}$ of the same detectors are also given in Table 1.

\section{Results}

We start our analysis by discussing the Ge detectors. Fig. 2 shows the differential rate on a Ge detector as a function of the electron-equivalent energy $E_{\mathrm{ee}}$. The solid lines refer to WIMPs of masses $m_{\chi}=60 \mathrm{GeV}$ (upper solid line) and $m_{X}=20 \mathrm{GeV}$ (lower solid line). For the upper line we used, as a reference value, $\sigma_{0}=10^{-9}$ nbarn and for the lower ones $\sigma_{0}=10^{-10}$ nbarn. The other parameters used in the calculation of the rates are $v_{\odot}=232 \mathrm{~km} \mathrm{sec}^{-1}, v_{\text {esc }}=650 \mathrm{~km} \mathrm{sec}^{-1}$ and $\rho_{X}=0.5 \mathrm{GeV} \mathrm{cm}{ }^{-3}$. The differential rates for a maximally co-rotating halo are plotted as dashed lines (the upper one refers to $m_{\chi}=60 \mathrm{GeV}$, the lower one to $m_{X}=20 \mathrm{GeV}$ ). The results for a counter-rotating halo are reported as dotted lines.

For low energies, the co-rotating model gives a larger rate as compared to the nonrotating model, since low values of $E_{\mathrm{ee}}$ mainly correspond to low WIMP velocities in the local frame, where the co-rotating DF is enhanced with respect to the nonrotating model, as is shown in Fig. 1. On the contrary, for higher values of $E_{\mathrm{ee}}$, the co-rotating rate becomes smaller than the nonrotating one, and the counter-rotation situation gives the highest rates. The difference among the three curves increases with the energy, the one corresponding to a co-rotating halo rapidly diverging from the other two. We also notice that, for a fixed value of $E_{\text {ee }}$, the difference between the rotating and nonrotating situations is more pronounced for lighter WIMPs. This property 


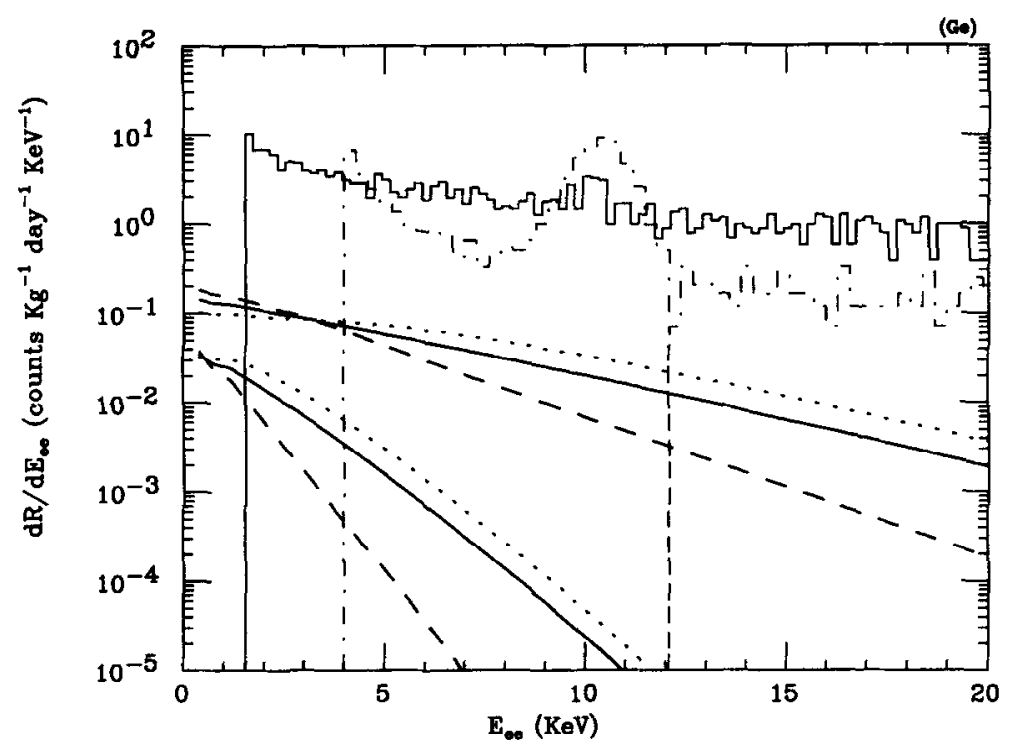

Fig. 2. Differential rate on a Ge detector as a function of the electron-equivalent energy $E_{\mathfrak{p e}}$. The solid, dashed and dotted lines refer respectively to the nonrotating, co-rotating and counter-rotating case. The upper lines refer to $m_{\chi}=60 \mathrm{GeV}$ and $\sigma_{0}=10^{-9}$ nbarn The lower curves are for $m_{\chi}=20 \mathrm{GeV}$ and $\sigma_{0}=10^{-10} \mathrm{nbarn}$. The solid (dot-dashed) histogram represents the $90 \%$ C.L. upper limit on the counting rate of the Neuchatel [12] (Twin [14]) experiment.

has a direct influence on the determination of the exclusion plot in the WIMP-nucleus cross section $\sigma_{0}$ vs $m_{\chi}$ plane, since the exclusion plot is obtained by comparing the calculated rate with the $90 \%$ C.L. upper limit on the counts of the detector. Therefore, because of a possible halo rotation, we expect a larger uncertainty for lighter WIMPs in the determination of the exclusion plots.

In Fig. 2 the $90 \%$ C.L. upper limits of two representative Ge experiments are also plotted: the solid histogram refers to the Neuchatel experiment [12], the dot-dashed histogram is obtained from the Twin experiment [14]. Because of the relatively fast decrease of the differential rates as a function of $E_{\mathrm{ee}}$, the most stringent limits on the WIMP parameters are usually obtained from the energy bins closest to threshold energy. For the Neuchatel experiment, the energy threshold of the detector is $1.5 \mathrm{keV}$. In the case of Twin, the detector energy threshold is $4 \mathrm{keV}$, but the most stringent limits are provided by the counting rates in the energy bins above the Gallium peak (which is clearly visible as an increase in the counting rate around $E_{\mathrm{ee}} \simeq 10 \mathrm{keV}$ ). This fact gives an effective threshold for WIMP searches of about $12 \mathrm{keV}$, denoted by a vertical dashed line, similar to the $\mathrm{Ge} /$ Heidelberg experiment [13]. Fig. 2 shows that for Ge experiments with a low energy threshold the effects of halo rotation are less important than for the case where the threshold energy is high. This is especially true for a counter-rotating halo and high WIMP masses.

We are now in the position of determining the upper limit on the WIMP-nucleus cross section as a function of $m_{X}$ by comparing the calculated and the experimental rates. Actually, the direct detection technique measures the product $\left(\rho_{X} \times \sigma_{0}\right)$ between the WIMP local density $\rho_{X}$ and the WIMP-nucleus cross section $\sigma_{0}$. The single parameters $\rho_{X}$ and $\sigma_{0}$ cannot be disentangled in a direct detection measurement. Therefore, we report our upper bounds in terms of the product $\xi \sigma$, where the WIMP local density is parametrized as a fraction $\xi$ of the local total dark matter density $\rho_{\odot}(\xi \leq 1)$ and we choose as a reference value $\rho_{\odot}=0.5 \mathrm{GeV} \mathrm{cm}^{-3}$. As it was discussed in Ref. [1], in the case of scalar coupling it is possible to report the results as upper limits on the WIMP-nucleon cross section (instead of a WIMP-nucleus one), which is more suitable for the comparison among different experiments, especially when they make use of different target nuclei. The WIMP-nucleon scalar cross section is defined as [1] 


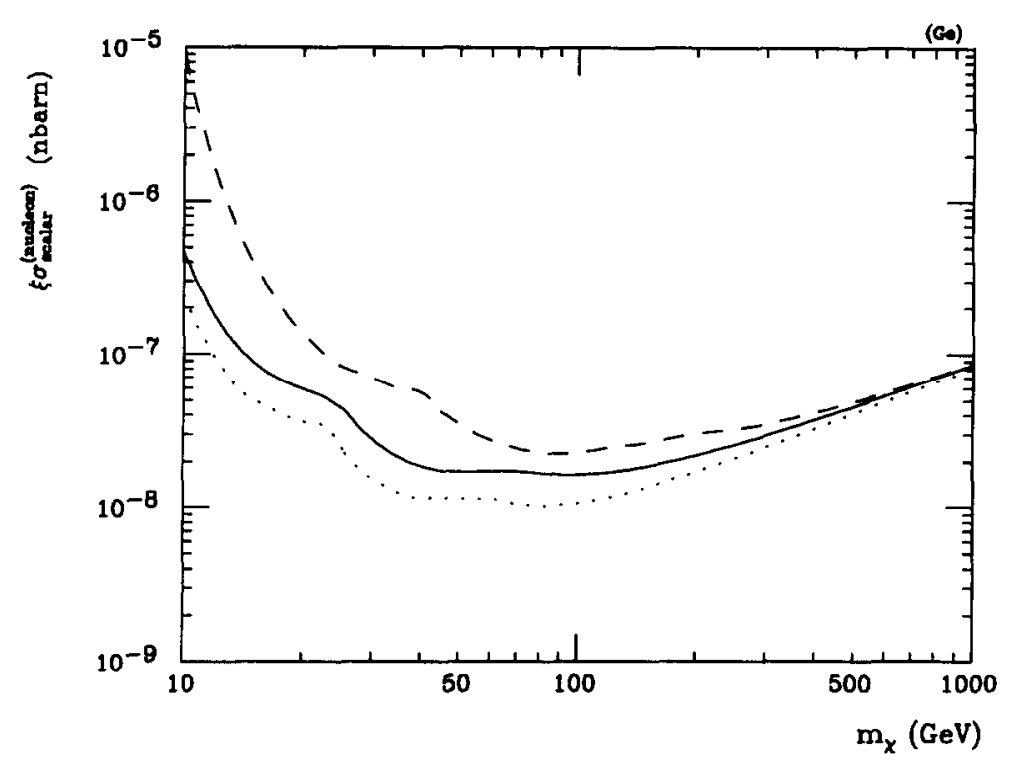

Fig. 3. The $90 \%$ C.L. upper limit on the quantity $\xi \sigma_{\text {scalar }}^{\text {(nucleon) }}$ as a function of the WIMP mass $m_{\chi}$ for the Ge detectors [12-15]. The solid, dashed and dotted lines refer to a nonrotating, co-rotating and counter-rotating halo, respectively.

$$
\sigma_{\text {scalar }}^{\text {(nucleon) }}=\left(\frac{1+m_{\chi} / m_{N}}{1+m_{\chi} / m_{p}}\right)^{2} \frac{\sigma_{0}}{A^{2}},
$$

where $m_{p}$ is the proton mass.

Fig. 3 shows the $90 \%$ C.L. upper limit on the quantity $\xi \sigma_{\text {scalar }}^{\text {(nuclen) }}$ as a function of the WIMP mass, for the case of a nonrotating halo (solid line), of maximal co-rotation (dashed line) and maximal counter-rotation (dotted line). The plot is the convolution of the most stringent limits of all the presently available results from Ge detectors [12-15]. In the WIMP mass range reported in Fig. 3, the upper limit for masses below 25 $\mathrm{GeV}$ (for nonrotating and counter-rotating halo models, $40 \mathrm{GeV}$ for co-rotation) is provided by the Neuchatel experiment and for higher masses the limit comes from Twin. Fig. 3 shows that the effect of rotation of the halo can strongly affect the low mass region in the co-rotating situation. The reason for this behaviour has been previously discussed in relation with Fig. 2, where it was shown that the detection rates for lighter WIMPs are more affected by a possible halo rotation. On the contrary, for masses above $50 \mathrm{GeV}$ the effect is contained below a factor of two, when the co-rotation case is compared to the nonrotating halo result. The difference between the two exclusion plots is smaller for heavier WIMPs. As reference values, the difference is of the order of $50 \%$ for masses around $100 \mathrm{GeV}$ and is reduced below $20 \%$ for $m_{\chi} \gtrsim 300 \mathrm{GeV}$. For counter-rotating models, the modification of the exclusion plot with respect to the nonrotating case is always relatively small, not exceeding a factor of two in the whole mass range. For WIMP masses above $100 \mathrm{GeV}$, the magnitude of the effect is very similar to the case of co-rotation. We have to remind at this point that the effect considered here refers to situations of maximal rotation of the galactic halo. Plausible models of rotating haloes lie somewhere in between the two maximal cases discussed here, probably much closer to the nonrotating case than to these extreme situations. Therefore, our results have to be considered as maximal possible effects of galactic rotation on the determination of the exclusion plots.

Let us now discuss the case of NaI detectors. The large mass low-background NaI detector of the DAMA/NaI Collaboration currently provides the most stringent upper limit on the WIMP-nucleon scalar cross section [16] (except for a narrow window around $m_{\chi} \simeq 15 \mathrm{GeV}$, where the Ge detectors are more sensitive [1].) Fig. 4 shows the differential rate for $\mathrm{NaI}$ detector as a function of the electron equivalent energy $E_{\text {ee. The solid line }}$ 


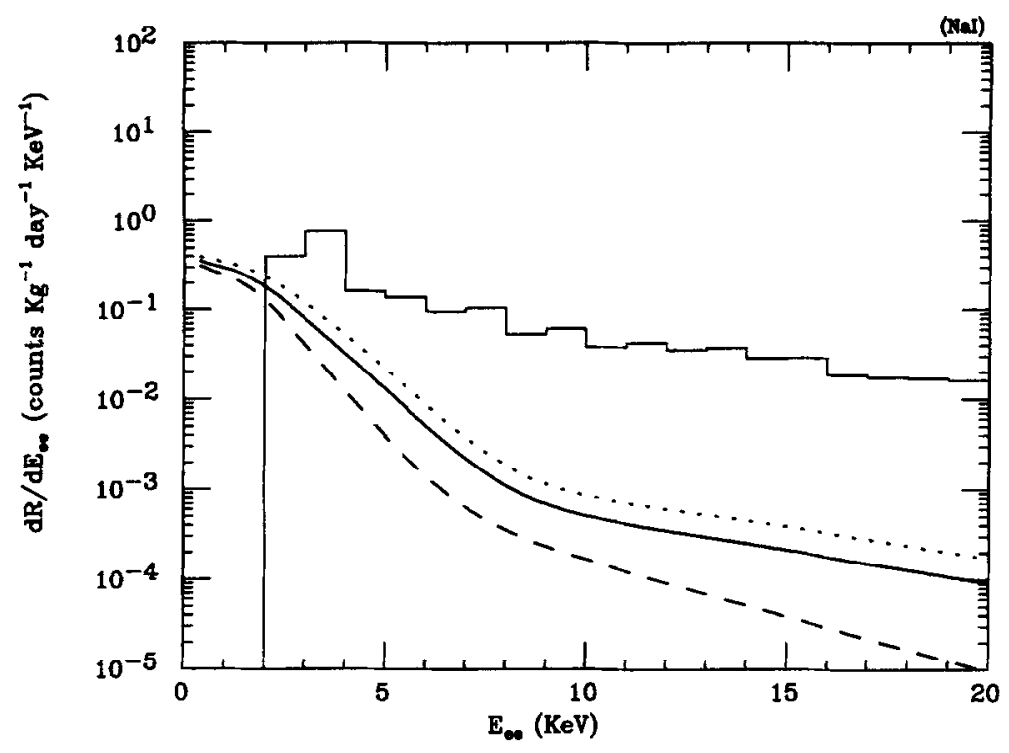

Fig. 4. Differential rate on a Nal detector as a function of the electron equivalent energy $E_{\mathrm{ee}}$, for a WIMP of $m_{X}=60 \mathrm{GeV}$ and $\sigma_{0}=10^{-9}$ nbarn. The solid, dashed and dotted lines refer to the nonrotating, co-rotating and counter-rotating case, respectively. The histogram represents the $90 \%$ C.L. upper limit on the counting rate obtained from the DAMA/NaI Collaboration [16].

is the rate calculated for $m_{\chi}=60 \mathrm{GeV}$ and $\sigma_{0}=10^{-9}$ nbarn in the case of a nonrotating halo. The dashed line refers to the co-rotating case and the dotted line to the counter-rotating situation. The histogram is the $90 \%$ C.L. upper limit from the DAMA/NaI Collaboration [16]. The ensuing exclusion plot is shown in Fig. 5. Also for the $\mathrm{NaI}$ detector, the co-rotating case deviates significantly from the nonrotating situation for relatively low masses. On the contrary, for counter-rotating haloes the exclusion plot remains close to the result for a static halo. The relative deviation $R=\left[\xi \sigma_{\text {scalar }}^{\text {(nucleon) }}\right]_{i} /\left[\xi \sigma_{\text {scallar }}^{\text {(nute })}\right]_{\text {nonrot }}$ ( $i$ stands for co- or counter-rotation) between the extracted upper limits in the case of rotation with respect to the nonrotating case, are shown in Fig. 6. The dashed (dotted) line refers to a co-rotating (counter-rotating) halo. We observe that also in this case, for WIMP masses larger that $50 \mathrm{GeV}$ the effect of rotation affects the exclusion plot by less than a factor of 2 . This effect is actually smaller for heavier WIMPs: for $m_{\chi} \gtrsim 80 \mathrm{GeV}$ the exclusion plot uncertainty is of the order of $20-30 \%$.

Finally, we show in Fig. 7 the effects of halo rotation on the exclusion plot for a ${ }^{129} \mathrm{Xe}$ detector. Due to the high nuclear mass, Xe detectors are in principle more sensitive to higher WIMP masses than the NaI and $\mathrm{Ge}$ ones. Moreover, the quenching factor of Xe detectors is larger than the one of Ge and NaI detectors, and this also shifts the sensitivity of Xe detectors to higher WIMP masses. The DAMA/Xe Collaboration recently reported the results of the analysis on an improved statistics of $1763.2 \mathrm{Kg} \times$ day obtained with an enriched liquid Xe scintillator [18]. The exclusion plot obtained from the data of Ref. [18] is plotted in Fig. 7, for the conservative value of $Q=0.44$. Similar to the cases previously discussed, for a counter-rotating halo the deviation is always smaller than a factor of two. The same situation happens for co-rotating models and WIMP masses larger than about $50 \mathrm{GeV}$. Regardless of the model of rotation, for $m_{\chi} \gtrsim 200 \mathrm{GeV}$ the deviation is always smaller than $25 \%$. 


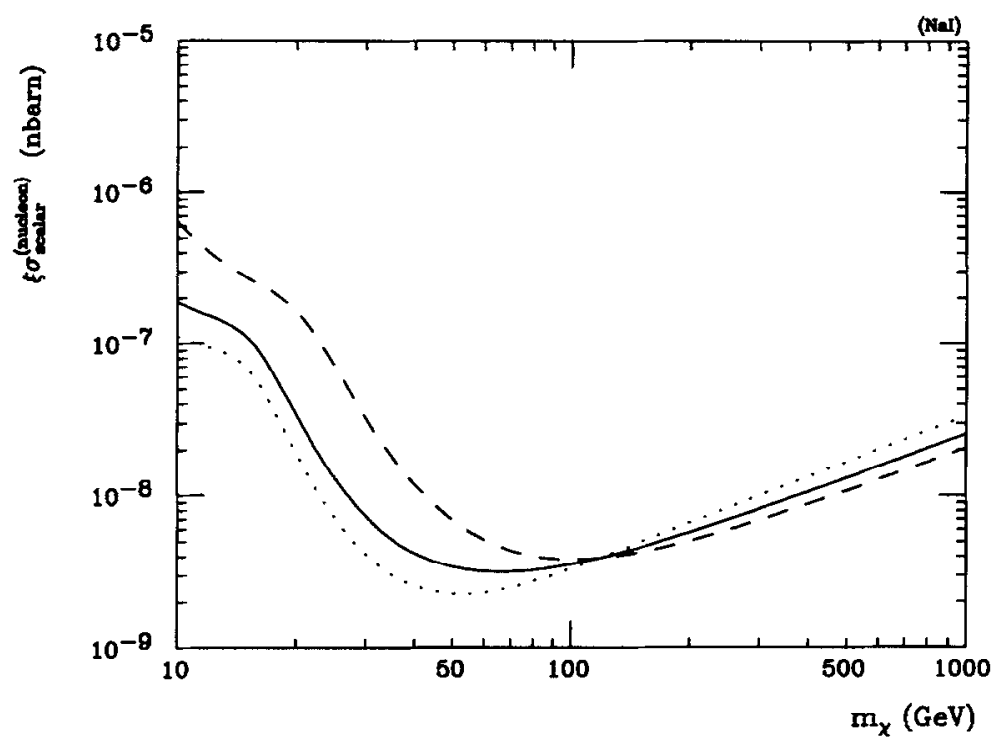

Fig. 5. The $90 \%$ C.L. upper limit on the quantity $\xi \sigma_{\text {scalar }}^{\text {(nucleon) }}$ as a function of the WIMP mass $m_{\chi}$ for the DAMA/Nal detector [16]. The solid, dashed and dotted lines refer to a nonrotating, co-rotating and counter-rotating halo, respectively.

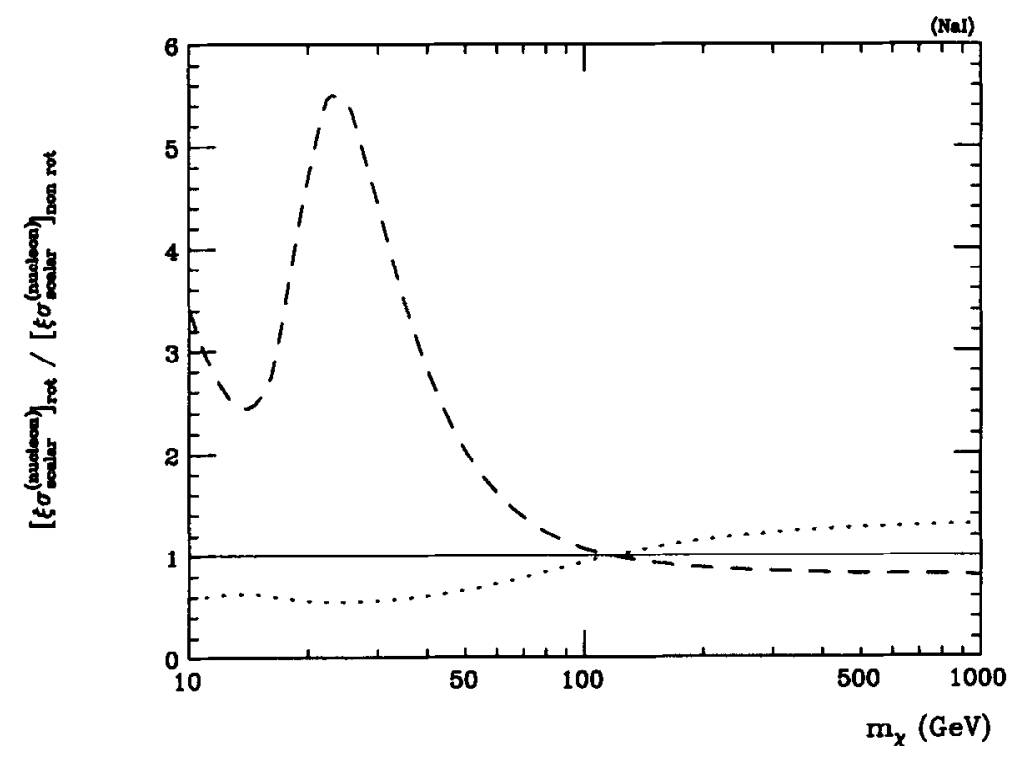

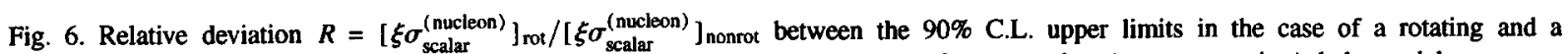
nonrotating halo, for the DAMA/Nal detector. The dashed (dotted) line refers to the co-rotating (counter-rotating) halo model.

\section{Conclusions}

In this paper we have investigated the effect induced by a possible rotation of the galactic halo on the rates of WIMP direct detection. In particular, we have discussed the implication of halo rotation on the determination of the exclusion plots on the WIMP-nucleon cross section for different detectors, namely Ge, $\mathrm{NaI}$ and Xe ones. The rotation of the halo has been described by using a model [10] which corresponds to a situation where the 


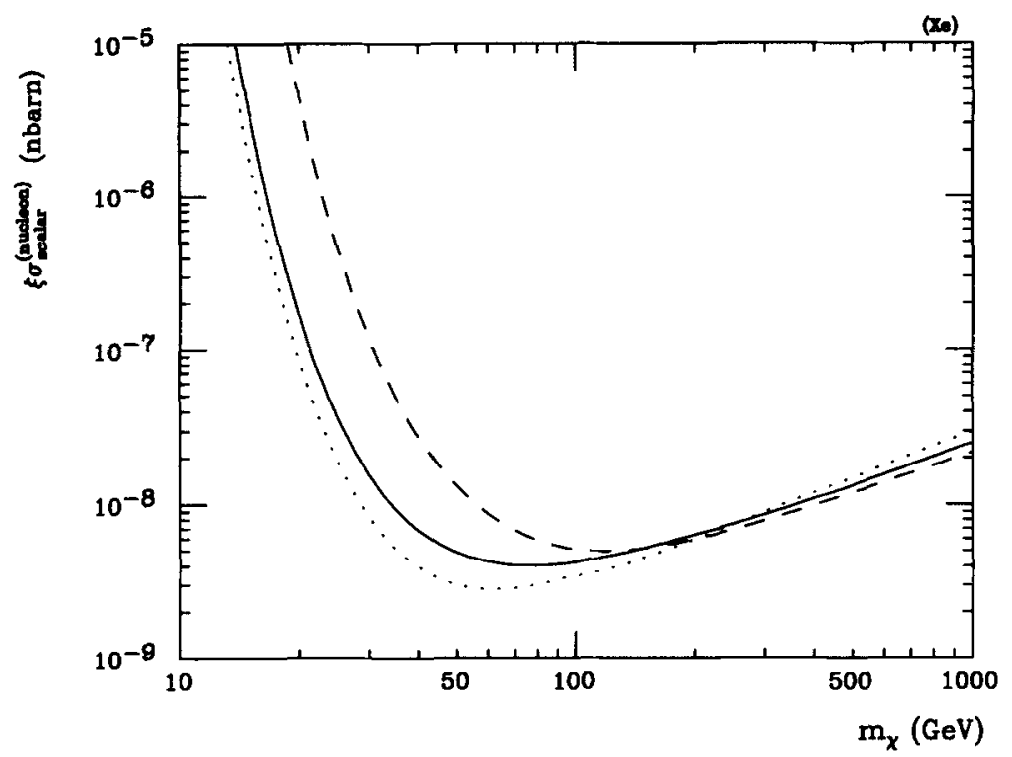

Fig. 7. The $90 \%$ C.L. upper limit on the quantity $\xi \sigma_{\text {scalar }}^{\text {(nucleon })}$ as a function of the WIMP mass $m_{\chi}$ for the DAMA/Xe detector [18]. The solid, dashed and dotted lines refer to a nonrotating, co-rotating and counter-rotating halo, respectively.

halo possesses the maximal rotation compatible with a given mass $\mathrm{DF}$, which for simplicity we have chosen to be that of the isothermal sphere.

We found that the exclusion plots obtained from the data are affected by less than a factor of 2 in the case of counter-rotating models. The same size of uncertainty occurs also for the co-rotating models, when the WIMP mass is larger than about $50 \mathrm{GeV}$. For lighter WIMPs and co-rotation, the exclusion plots are modified by a larger amount. We have to remind that, due to the particular model of halo rotation which we have employed here, these are expected to be maximal effects. For specific physical rotation models, the effect of halo rotation will be plausibly smaller. We can therefore conclude that, at least for WIMP masses greater than about 50 $\mathrm{GeV}$, the determination of the exclusion plots from the experimental data are affected by an uncertainty smaller than a factor of two due to the possibility that the galactic halo rotates, independently on the specific model of halo rotation. We notice that recent preliminary data from accelerators indicate that the lower limit on the mass of the most plausible WIMP candidate, the neutralino, is $m_{x} \simeq 30 \mathrm{GeV}$ for low value of the susy parameter $\tan \beta$, and $m_{x} \simeq 45 \mathrm{GeV}$ for $\tan \beta \gtrsim 3$ [27]. Therefore, for this dark matter candidate, the uncertainty on the exclusion plot due to a possible rotation of the halo is expected to be relatively small. The situation is different for lighter WIMPs. In this case, it would be required to develop specific models of halo rotation in order to obtain more accurate conclusions.

\section{Acknowledgements}

We wish to thank Sandro Bottino for many useful discussions and encouragement in the preparation of this paper. We also like to thank Rita Bernabei for illuminating discussions about the direct detection technique. 


\section{Appendix A}

In this appendix we report the analytical expression of the function $\mathcal{I}\left(v_{\min }, v_{\odot}, v_{\text {esc }}\right)$ which enters in the calculation of the differential rate for direct detection equation (6), for a velocity DF which is Maxwellian in the galactic frame (see Eq. (4)). The cases of a nonrotating, maximally co-rotating and maximally counterrotating halo models are given. In all the following expressions we make use of the dimensionless variables $x^{2}=3 v^{2} /\left(2 w^{2}\right), \eta^{2}=3 v_{\odot}^{2} /\left(2 w^{2}\right), z^{2}=3 v_{\mathrm{esc}}^{2} /\left(2 w^{2}\right), x_{\min }=\left(3 m E_{R} / 4 m_{\mathrm{red}}^{2} w^{2}\right)^{1 / 2}$, where $v_{\odot}$ denotes the Earth velocity in the galactic frame, $v_{\text {esc }}$ is the escape velocity, $w$ is the root mean square velocity of the Maxwellian distribution in the galactic rest frame. In the definition of the three distributions, the same value of $w$ has to be used, since they correspond to the same matter density and therefore they contribute in the same way to the rotational velocities. We also define the function

$$
\chi(x, y)=\frac{\sqrt{\pi}}{2}[\operatorname{erf}(y)-\operatorname{erf}(x)],
$$

where

$$
\operatorname{erf}(x)=\frac{2}{\sqrt{\pi}} \int_{0}^{x} \exp \left(-t^{2}\right) d t
$$

The normalization constant $N$ of the Maxwellian DF is given in Eq. (5).

\section{A.1. Nonrotating model}

$$
\begin{aligned}
& \mathcal{I}\left(v_{\min }, v_{\odot}, v_{\mathrm{esc}}\right)=\frac{N}{\eta}\left(\frac{3}{2 \pi w^{2}}\right)^{1 / 2} \\
& \quad \times \begin{cases}\chi\left(x_{\min }-\eta, x_{\min }+\eta\right)-2 \eta \exp \left(-z^{2}\right) & x_{\min }<z-\eta, \\
\chi\left(x_{\min }-\eta, z\right)-\exp \left(-z^{2}\right)\left(z+\eta-x_{\min }\right) & z-\eta \leq x_{\min }<z+\eta, \\
0 & x_{\min } \geq z+\eta .\end{cases}
\end{aligned}
$$

\section{A.2. Maximally co-rotating Lynden-Bell model}

In this case there are two possible situations, depending on the relative magnitude of $v_{\odot}$ and $v_{\text {esc }}$.

(i) $2 \eta \leq z$

$$
\begin{aligned}
& \mathcal{I}\left(v_{\min }, v_{\odot}, v_{\text {esc }}\right)=2 \frac{N}{\eta}\left(\frac{3}{2 \pi w^{2}}\right)^{1 / 2} \\
& \quad \times \begin{cases}\chi\left(x_{\min }-\eta, x_{\min }+\eta\right)+\chi(z, 0)+\exp \left(\eta^{2}\right) \chi\left(\eta, \sqrt{z^{2}+\eta^{2}}\right) & x_{\min } \leq \eta, \\
\quad-\exp \left(-z^{2}\right)\left(\eta-z+\sqrt{z^{2}+\eta^{2}}\right) & \\
\chi\left(z, x_{\min }+\eta\right)+\exp \left(\eta^{2}\right) \chi\left(x_{\min }, \sqrt{z^{2}+\eta^{2}}\right) & \eta<x_{\min } \leq z-\eta, \\
-\exp \left(-z^{2}\right)\left(\eta-z+\sqrt{z^{2}+\eta^{2}}\right) & z-\eta<x_{\min } \leq \sqrt{z^{2}+\eta^{2}}, \\
\exp \left(\eta^{2}\right) \chi\left(x_{\min }, \sqrt{z^{2}+\eta^{2}}\right)-\exp \left(-z^{2}\right)\left(\sqrt{z^{2}+\eta^{2}}-x_{\min }\right) & x_{\min } \geq \sqrt{z^{2}+\eta^{2}} . \\
0 & \end{cases}
\end{aligned}
$$


(ii) $2 \eta \geq z$

$$
\begin{aligned}
& \mathcal{I}\left(v_{\text {nin }}, v_{\odot}, v_{\text {esc }}\right)=2 \frac{N}{\eta}\left(\frac{3}{2 \pi w^{2}}\right)^{1 / 2} \\
& \quad \times \begin{cases}\chi\left(x_{\min }-\eta, x_{\min }+\eta\right)+\chi(z, 0)+\exp \left(\eta^{2}\right) \chi\left(\eta, \sqrt{z^{2}+\eta^{2}}\right) & x_{\min } \leq z-\eta, \\
\quad-\exp \left(-z^{2}\right)\left(\eta-z+\sqrt{z^{2}+\eta^{2}}\right) & \\
\chi\left(x_{\text {nui }}-\eta, 0\right)+\exp \left(\eta^{2}\right) \chi\left(\eta, \sqrt{z^{2}+\eta^{2}}\right) & z-\eta<x_{\min } \leq \eta \\
-\exp \left(-z^{2}\right)\left(\sqrt{z^{2}+\eta^{2}}-x_{\min }\right) & \eta<x_{\min } \leq \sqrt{z^{2}+\eta^{2}}, \\
\exp \left(\eta^{2}\right) \chi\left(x_{\min }, \sqrt{z^{2}+\eta^{2}}\right)-\exp \left(-z^{2}\right)\left(\sqrt{z^{2}+\eta^{2}}-x_{\min }\right) & x_{\min } \geq \sqrt{z^{2}+\eta^{2}} \\
0 & \end{cases}
\end{aligned}
$$

\section{A.3. Maximally counter-rotating Lynden-Bell model}

$$
\begin{aligned}
& I\left(v_{\min }, v_{\odot}, v_{\mathrm{esc}}\right)=2 \frac{N}{\eta}\left(\frac{3}{2 \pi w^{2}}\right)^{1 / 2} \\
& \quad \times \begin{cases}\chi(0, z)+\exp \left(\eta^{2}\right) \chi\left(\sqrt{z^{2}+\eta^{2}}, \eta\right) & x_{\min } \leq \eta, \\
-\exp \left(-z^{2}\right)\left(\eta+z-\sqrt{z^{2}+\eta^{2}}\right) & \\
\chi\left(x_{\min }-\eta, z\right)+\exp \left(\eta^{2}\right) \chi\left(\sqrt{z^{2}+\eta^{2}}, x_{\min }\right) & \\
-\exp \left(-z^{2}\right)\left(\eta+z-\sqrt{z^{2}+\eta^{2}}\right) & \eta<x_{\min } \leq \sqrt{z^{2}+\eta^{2}}, \\
\chi\left(x_{\min }-\eta, z\right)-\exp \left(-z^{2}\right)\left(z+\eta-x_{\min }\right) & \sqrt{z^{2}+\eta^{2}}<x_{\min } \leq z+\eta, \\
0 & x_{\min } \geq z+\eta .\end{cases}
\end{aligned}
$$

\section{References}

[1] A. Bottino, F. Donato, G. Mignola, S. Scopel, P. Belli, A. Incicchitti, Phys. Lett. B 402 (1997) 113;

N. Fornengo, Proc. Int. Workshop on Physics Beyond the Standard Model: from theory to experiment (Valencia97), Valencia, October 1997; Proc. Workshop on DM-Italia 97: Dark matter: perspectives and projects. Trieste. December 1997.

[2] D. Lynden-Bell, MNRAS 136 (1967) 101.

[3] J. Binney, S. Tremaine, Galactic Dynamics (Princeton Univ. Press, Princeton, 1987).

[4] E.I. Gates, G. Gyuk, M.S. Turner, Ap. J. 449 (1995) L123.

[5] N.W. Evans, MNRAS 260 (1993) 191.

[6] A. Bottino, C. Favero, N. Fornengo, G. Mignola, Astrop. Phys. 7 (1995) 73.

[7] T. Mitsui, K. Maki, S. Orito, Phys. Lett. B 389 (1996) 169.

[8] L. Bergström, P. Ullio, J.H. Buckley, astro-ph/9712318, December 1997.

[9] M. Kamionkowski, A. Kinkhabwala, hep-ph/9710337, October 1997.

[10] D. Lynden-Bell, MNRAS 120 (1960) 204.

[11] S.P. Ahlen et al, Phys. Let. B 195 (1987) 603;

D.O. Caldwell et al., Phys. Rev. Lett. 61 (1988) 510.

[12] D. Reusser et al., Phys. Lett. B 255 (1991) 143.

[13] M. Beck, Heidelberg-Moscow Collaboration, Nucl. Phys. B (Proc. Suppl.) 35 (1994) 150; M. Beck et al., Phys. Lett. B 336 (1994) 141.

[14] A.K. Drukier et al., Nucl. Phys. B (Proc. Suppl.) 28A (1992) 293;

I.R. Sagdev, A.K. Drukier, D.J. Welsh, A.A. Klimenko, S.B. Osetrov, A.A. Smolnikov, Nucl. Phys. B (Proc. Suppl.) 35 (1994) 175

[15] E. Garcia et al., Nucl. Phys. B (Proc. Suppl.) 28A (1992) 286; M.L. Sarsa et al., Nucl. Phys. B (Proc. Suppl.) 35 (1994) 154; 
E. Garcia et al., Proc. "The Dark Side of the Universe", Rome 1994, R. Bernabei, C. Tao, eds. (World Scientific, Singapore, 1994) p. 216.

[16] R. Bernabei et al., Phys. Lett. B 389 (1996) 757.

[17] P.F. Smith et al., Phys. Lett. B 379 (1996) 299.

[18] R. Bernabei et al., ROM2F/98/08, February 1998.

[19] R. Bernabei et al., Astrop. Phys. 7 (1997) 73.

[20] A. Alessandrello et al., Phys. Lett. B 384 (1996) 316.

[21] A. Bottino, F. Donato, N. Fornengo, S. Scopel, DFTT 49/97, hep-ph/9709292, Phys. Lett. B, to appear.

[22] R.H. Helm, Phys. Rev. D 104 (1956) 1466; J. Engel, Phys. Lett. B 264 (1991) 114.

[23] F.J. Kerr, D. Lynden-Bell, MNRAS 221 (1986) 1023.

[24] P.J.T, Leonard, S. Tremaine, Ap. J. 353 (1990) 486.

[25] P.F. Smith, J.D. Lewin, Phys. Rep. 187 (1990) 203.

[26] J. Barnes, G. Efstathiou, Ap. J. 319 (1987) 575;

M.S. Warren, P.J. Quinn, J.K. Salmon, W.H. Zurek, Ap. J. 399 (1992) 405.

[27] P. Dornan, ALEPH Collaboration, presented at LEPC Conference, November 1997;

P. Charpentier, DELPHI Collaboration, presented at LEPC Conference, November 1997;

M. Pohl, L3 Collaboration, presented at LEPC Conference, November 1997;

A. Honma, OPAL Collaboration, presented at LEPC Conference, November 1997. 